\title{
EMBEDDING OF COMPLETE MOORE SPACES
}

\author{
GEOFFREY D. CREEDE ${ }^{1}$
}

Abstract. The purpose of this paper is to give necessary and sufficient conditions for Moore spaces to be complete in terms of embeddings in bicompact spaces.

In [2], Cech showed that a metric space $X$ is complete if and only if there exists a bicompact Hausdorff space $Y \supset X$ such that $X$ is $G_{\delta}$ in $Y$. We shall show that this result can be extended to completely regular Moore spaces. It is also shown that the condition of complete regularity can be eliminated by replacing Hausdorff with $T_{1}$ and placing additional conditions on the embedding of $X$ in $Y$.

Some of the results of this paper were announced in [3].

Terms which are not defined within are used as in [4].

Definition 1. A sequence $\left\{g_{i}\right\}_{i=1}^{\infty}$ of open covers of a topological space $X$ is a development for $X$ if (1) $\mathcal{G}_{i+1}$ is a refinement for $\mathcal{G}_{i}$, and (2) if $x$ is a point of $X$ and $U$ is an open set in $X$ containing $x$, then there is a natural number $k$ such that $\operatorname{St}\left(x, \varsigma_{k}\right) \subset U$. A Moore space is a regular $T_{1}$-space which has a development. A Moore space is complete with respect to the development $\left\{g_{n}\right\}_{n=1}^{\infty}$ provided that, if $\left\{A_{i}\right\}_{i=1}^{\infty}$ is a nonincreasing sequence of nonempty closed sets such that, for each $n$, there is a $G_{n} \in \mathcal{G}_{n}$ with $A_{n} \subset G_{n}$, then $\bigcap_{i=1}^{\infty} A_{i} \neq \varnothing$. A Moore space is complete if it has a development with respect to which it is complete. See [5].

Definition 2. A subspace $X$ of a topological space $Y$ is regularly embedded in $Y$ provided that if $p$ is a point of $X$ and $U$ is an open subset of $Y$ containing $p$, then there is an open subset $V$ of $Y$ containing $p$ whose closure is contained in $U$.

It should be noticed here that any subspace of a regular space is regularly embedded in it and, if $X$ is regularly embedded in $Y$, then $X$ is regular. However, neither of these two implications can be reversed.

TheOREM 3. Let $X$ be a Moore space. If there is a bicompact space $Y$

Received by the editors August 17, 1969 .

A MS 1969 subject classifications. Primary 5438.

Key words and phrases. Complete Moore space, development, Wallman compactification, Stone-Čech compactification.

1 This paper is part of the author's doctoral dissertation written under the direction of R. W. Heath. The research was supported by a NASA Traineeship and a NSF Fellowship. 
in which $X$ is regularly embedded such that $X$ is a $G_{\delta}$ subset of $Y$, then $X$ is complete.

Proof. Let $\left\{g_{i}\right\}_{i=1}^{\infty}$ be a development for $X$ and let $\left\{P_{i}\right\}_{i=1}^{\infty}$ be a sequence of open subsets of $Y$ such that $X=\bigcap_{i=1}^{\infty} P_{i}$. For each natural number $i$ and each point $x$ in $X$, let $G_{i}(x)$ be an open subset of $Y$ containing $x$ such that $G_{i}(x) \cap X \in \mathcal{G}_{i}$. For each $x \in X$, let $\left\{H_{i}(x)\right\}_{i=1}^{\infty}$ be a sequence of open subsets of $Y$ each of which contains $x$ such that, for each $i, \mathrm{Cl}_{Y} H_{i}(x) \subset G_{i}(x) \cap P_{i}$ and $H_{i+1}(x) \subset H_{i}(x)$. Let $\mathfrak{F C}_{i}=\left\{H_{i}(x) \cap X: x \in X\right\}$. Since $\mathfrak{F C}_{i+1}$ refines $\mathfrak{F C}_{i}$ and $\mathfrak{F C}_{i}$ refines $\mathcal{G}_{i}$, the sequence $\left\{\mathcal{K}_{i}\right\}_{i=1}^{\infty}$ is a development for $X$. Let $\left\{A_{i}\right\}_{i=1}^{\infty}$ be a nonincreasing sequence of nonempty closed subsets of $X$ such that, for each $i$, there is a $H_{i}^{\prime} \in \mathcal{F}_{i}$ such that $A_{i} \subset H_{i}^{\prime}$. The sequence $\left\{\mathrm{Cl}_{Y} A_{i}\right\}_{i=1}^{\infty}$ is a nonincreasing sequence of nonempty bicompact sets and, hence, $\bigcap_{i=1}^{\infty} \mathrm{Cl}_{Y} A_{i} \neq \varnothing$. But, for each $i, \mathrm{Cl}_{Y} A_{i} \subset \mathrm{Cl}_{Y} H_{i}^{\prime} \subset P_{i}$. Thus, $\bigcap_{i=1}^{\infty} A_{i}$ $=\bigcap_{i=1}^{\infty} \mathrm{Cl}_{Y} A_{i} \neq \varnothing$. Therefore, $X$ is complete with respect to the development $\left\{\mathfrak{H}_{i}\right\}_{i=1}^{\infty}$.

TheOREM 4. A complete Moore space is a $G_{\delta}$ subset of every $T_{1}$-space in which it is dense and regularly embedded.

Proof. Let $X$ be a Moore space which is complete with respect to the development $\left\{g_{i}\right\}_{i=1}^{\infty}$. Let $X$ be dense and regularly embedded in the $T_{1}$-space $Y$. For each natural number $i$ and each point $x$ in $X$, let $G_{i}(x)$ be an open subset of $Y$ containing $x$ such that $G_{i}(x) \cap X \in \mathcal{G}_{i}$ and $H_{i}(x)$ be an open subset of $Y$ containing $x$ such that $\mathrm{Cl}_{Y} H_{i}(x)$ $\subset G_{i}(x)$. For each $i$, let $P_{i}=\bigcup\left\{H_{i}(x): x \in X\right\}$. It is evident that $X \subset \cap_{i=1}^{\infty} P_{i}$. Let $p \in Y-X$ and suppose that $p \in \bigcap_{i=1}^{\infty} P_{i}$. For each $i$, there is a point $x_{i} \in X$ such that $p \in H_{i}\left(x_{i}\right)$. For each $n$, let $A_{n}$ $=\left(\bigcap_{i=1}^{n} \mathrm{Cl}_{Y} H_{i}\left(x_{i}\right)\right) \cap X$. Since $X$ is dense in $Y$, each $A_{n}$ is nonempty. Thus, $\left\{A_{i}\right\}_{i=1}^{\infty}$ is a nondecreasing sequence of nonempty closed sets in $X$ such that, for each $i, A_{i} \subset G_{n}\left(x_{n}\right)$. Since $X$ is complete with respect to the development $\left\{g_{i}\right\}_{i=1}^{\infty}$, there is a point $q$ common to all the $A_{i}$. Since $X$ is regularly embedded in the $T_{1}$-space $Y$, there is an open subset $U$ of $Y$ containing $q$ with $p \notin \mathrm{Cl}_{Y} U$. There is a natural number $k$ such that, if $q \in G$ and $G \in \mathcal{S}_{k}$, then $G \subset U \cap X$. Since $X$ is dense in $Y, H_{k}\left(x_{k}\right) \subset \mathrm{Cl}_{Y}\left(H_{k}\left(x_{k}\right) \cap X\right)$ and $H_{k}\left(x_{k}\right) \subset \mathrm{Cl}_{Y} U$. Thus, $p \notin H_{k}\left(x_{k}\right)$ and $X=\bigcap_{i=1}^{\infty} P_{i}$.

Theorem 4 can be strengthened by replacing the requirement that $X$ be dense in $Y$ with $\mathrm{Cl}_{Y} X$ is a $G_{\delta}$ subset of $Y$.

Any completely regular space is dense and, by the remark following Definition 2, regularly embedded in its Stone-Cech compactification. Hence, Theorems 3 and 4 have the following corollary. 
Corollary 5. A completely regular Moore space $X$ is complete if and only if there is a bicompact Hausdorff space $Y \supset X$ such that $X$ is a $G_{\delta}$ subspace of $Y$.

If $X$ is a $T_{1}$-space, let $w X$ denote its Wallman compactification [1], [4], [7]. In [7], it is proved that $w X$ is a bicompact $T_{1}$-space, but is not Hausdorff unless $X$ is normal. We will show that, if $X$ is regular, then $X$ is regularly embedded in $w X$. Hence, any nonnormal $T_{3}$-space and its Wallman compactification is an example of a space which is regularly embedded in a nonregular space.

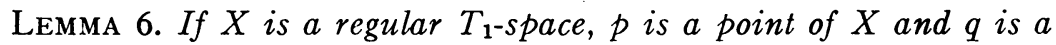
point of $w X$ distinct from $p$, then there are two disjoint open subsets of $w X$ separating $p$ and $q$.

Proof. Let $Q$ be the ultrafilter of closed sets in $X$ which corresponds to $q$. Since $p$ and $q$ are distinct, there is a closed set $Q \in Q$ which does not contain $p$. Since $X$ is regular, there are disjoint open subsets $U$ and $V$ of $X$ containing $p$ and $Q$, respectively. The open subsets $v(U)$ and $v(V)$ of $w X$ induced by $U$ and $V$, respectively, are disjoint and contain $p$ and $q$, respectively.

Theorem 7. A regular space is regularly embedded in its Wallman compactification.

Theorem 7 follows from the previous lemma by a modification of the proof that a bicompact Hausdorff space is regular.

Thus, by using the Wallman compactification instead of the StoneCech compactification we can remove the condition that the space be completely regular in Corollary 5 .

Theorem 8. A Moore space is complete if and only if there is a bicompact $T_{1}$-space $Y$ such that $X$ is regularly embedded in $Y$ and $X$ is a $G_{\delta}$ subset of $Y$.

Theorem 8 is a significant extension of Corollary 5 , since there do exist Moore spaces which are not completely regular. In fact, there are examples of complete Moore spaces whose only real valued continuous functions are constant functions [6], [8].

\section{REFERENCES}

1. R. M. Brooks, On Wallman compactifications, Fund. Math. 60 (1967), 157-173.

2. Eduard Čech, On bicompact spaces, Ann. of Math. 38 (1937), 823-844.

3. G. D. Creede, Semi-stratifiable spaces, Topology Conference, Arizona State University (1967), Tempe, Ariz., 1968, pp. 318-323. 
4. J. L. Kelley, General topology, Van Nostrand, Princeton, N. J., 1955. MR 16, 1136.

5. R. L. Moore, Foundations of point set theory, Amer. Math. Soc. Colloq. Publ., vol. 13, Amer. Math. Soc., Providence, R. I., 1932; rev. ed., 1962. MR 27 \#09.

6. Prabir Roy, Dual of a Moore space, Notices Amer. Math. Soc. 9 (1962), 327-328. Abstract \#62T-219.

7. Henry Wallman, Lattices and topological spaces, Ann. of Math. 39 (1938), 112126.

8. J. N. Younglove. A locally connected, complete Moore space on which every real valued continuous function is constant, Proc. Amer. Math. Soc. 20 (1969), 527-530. MR 40 \#1992.

Arizona State University, Tempe, Arizona 85281

Louisiana State, University, Baton Rouge, Lovisiana 70803 